\title{
Diseño e implementación de un instrumento electrónico de medida de PH para terreno agrícola
}

\section{Determination Design and Implementation of an electronic device for measuring $\mathrm{pH}$ in agricultural grounds}

\author{
Noel Jair Zambrano Sánchez (iD), Elber Fernando Camelo Quintero (iD), \\ Alejandro Méndez González (iD), Yeison Edward Valderrama Lugo \\ Universidad Nacional Abierta y a Distancia - UNAD, Colombia
}

Open Access

Recibido:

4 de abril de 2018

Aceptado:

19 de julio de 2018

Publicado:

11 diciembre de 2018

Correspondencia:

Noel.zambrano@unad.edu.co,

Elber.camelo@unad.edu.co,

yeison787@gmail.com

DOI:

https://doi.org/10.17081/invinno.7.1 .3042

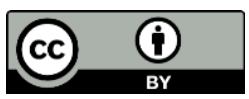

(c) Copyright: Investigación e Innovación en Ingenierías

\begin{abstract}
Resumen
Objetivo: Diseñar un instrumento de medida de $\mathrm{pH}$ que permita monitorear las variables al momento de iniciar un proceso agrícola. Metodología: Se utilizó la tecnología aplicada, que permitió brindar solución a una necesidad agrícola mediante la aplicación de competencias en las áreas de la ingeniería electrónica y la química, proceso que se desarrolló en cuatro fases. Resultados y conclusiones: $\mathrm{E}$ prototipo de $\mathrm{pH}$-metro desarrollado se acerca a las expectativas de diseño que se plantearon en un principio, pues se obtuvo una disminución notoria en los costos de implementación del prototipo (alrededor del 50\%) respecto a los $\mathrm{pH}$-metros usados para realizar las pruebas, lo que garantizó confiabilidad en la medición.
\end{abstract}

Palabras claves: pH, agricultura, procesador, amplificación, escalización.

Abstract

Objective: To design a pH measuring device capable to monitor the different variables at the moment of initiating any agricultural process. Methodology: It was used an applied technology that allowed to bring a solution to the needs in the agricultural area, through the application of competences in the grounds of electronic engineering and chemistry. This process was developed in 4 stages. Results y Conclusions: the prototype of the developed $\mathrm{pH}$-meter is close to the design expectations originally presented, obtaining a notorious reduction on the prototype implementation costs (around 50\%) in relation to the pH -meters utilized in the tests, thus guaranteeing reliability in the measurement.

Keywords: pH, Agriculture, Processor, Amplification, Scaled weighting.

Como citar (IEEE): N. Zambrano Sánchez, E. Camelo Quintero, A. Méndez González, y Y. Valderrama Lugo, “Diseño e implementación de un instrumento electrónico de medida de $\mathrm{PH}$ para terreno agrícola", Revista Investigación e Innovación en Ingenierías, vol. 7, n. 1, 2019. DOI: https://doi.org/10.17081/invinno.7.1.3042 


\section{Introducción}

Desde que el bioquímico Sören Sörensen inventó la escala de pH en 1909, esta variable se ha tenido en cuenta en distintos ámbitos, siendo el control de suelos para la agricultura uno de los más importantes [1].

En este sentido, es fundamental conocer los niveles de $\mathrm{pH}$ de las tierras, ya que afectan los cultivos de dos maneras: la primera proviene de los $\mathrm{pH}$ extremos que inciden en la disponibilidad de nutrientes necesarios para el crecimiento de la planta; la segunda, de complicaciones que surgen durante el proceso de absorción por parte de las raíces de la planta, que puede afectarlas hasta el punto de toxicidad, como en el caso del aluminio [2, 3].

Ahora bien, las diferentes especies de plantas necesitan nutrientes del suelo en diversas cantidades. En algunos casos, según el estado del suelo donde se realizará el cultivo, es necesario aplicar agro-insumos o fertilizantes a fin de alcanzar el rango óptimo de pH para el desarrollo de la planta [4]. Esto se debe a que el nivel de $\mathrm{pH}$ determina la acidez o alcalinidad del suelo, que afecta directamente a la planta en la obtención de minerales $[5,6]$.

En la industria, hay gran cantidad de medidores de $\mathrm{pH}$ para diferentes usos y su costo puede oscilar entre $\$ 800.000$ y $\$ 7^{\prime} 500.000$, y los más comunes se encuentran en laboratorios donde realizan mediciones sobre soluciones líquidas. Los más utilizados se emplean en la medición de pH en acuarios, piscinas y aguas de riego, que requieren de test y kits necesarios para su calibración en cada medición; tenemos en concreto los sensores de tipo Medidor de pH Adwa Ad11, con un costo de 32 euros para aplicaciones como las antes mencionadas; otro tipo de pH-metro es el Vanguard Hidroponics, a un costo de 24 euros, especial para medición de acuarios y cultivos hidropónicos con una gran prestigio pero no apto para el trabajo en campo [6].

También existen en el mercado instrumentos de alta tecnología para medición de $\mathrm{pH}$ en suelos, como el medidor IQ 150 con el tipo de sonda ISFET, una alta resolución de 0.01 rango de 0 a $14 \mathrm{pH}$ y alta resistencia a la temperatura $\left(105^{\circ} \mathrm{C}\right)$. El inconveniente es su elevado precio de $1.384,24$ euros más gastos de envió, muy alto para ser asumido por un agricultor o agrónomo, siendo este el que más se ajusta a la necesidad de medición in situ [7].

El pH es una variable difícil de validar y medir en terreno. Antes se debían tomar las muestras y llevarlas a un laboratorio, donde hacían los análisis y días después se entregaban los resultados. Por todo ello, viendo la necesidad de desarrollar una herramienta que dé al agricultor o agrónomo la ventaja de tener un instrumento de medición de $\mathrm{pH}$ para terrenos cultivables que reúna las características fundaméntales de precisión, medición in situ y bajo 
costo [8], se diseñó e implementó un instrumento digital para mediciones precisas y confiables de $\mathrm{pH}$ en el rango utilizado en suelos agrícolas (escala de 4 a 8). Como cuenta con una precisión y repetitividad dentro del rango de aceptación y un bajo precio, resulta una solución viable como equipo de medición en campo o su implementación en un proyecto de robótica agrícola [9].

\section{Justificación}

Los agricultores deben mejorar permanentemente los procesos, y así garantizar la calidad de los productos alimenticios que presentarán a los consumidores [2]. Ofrecer herramientas tecnológicas que les permitan mejorar sus prácticas agrícolas mediante la medición in situ de variables directamente sobre el suelo, y no esperando que la planta presente signos adversos o unos resultados de laboratorio semanas después de tomadas las muestras, les permite tomar acciones inmediatas $y$, en consecuencia, la posibilidad de satisfacer el mercado con un producto nutritivo y saludable $[10,11]$.

Es aquí donde se justifica la importancia del medidor de $\mathrm{pH}$, ya que la precisión dada por este instrumento electrónico le permitirá al agricultor tomar decisiones frente a su cultivo, que le permitan mantenerlo en un suelo con un $\mathrm{pH}$ controlado y óptimo para su desarrollo. Otro aspecto importante en este proyecto es el costo del instrumento de medición de $\mathrm{pH}$, ya que se busca ofrecer una herramienta de bajo costo que pueda servir al agricultor, agrónomo o aficionado al agro; es decir un instrumento de medición de $\mathrm{pH}$ directo al suelo, de costo módico y con características de un medidor de $\mathrm{pH}$ de las marcas más reconocidas, en si una herramienta idóneo para suelos al alcance del agricultor de nuestra región. [2,9]

\section{Metodología}

\section{Instrumento para Medición de PH}

Tipo de investigación: Aplicada Tecnológica

Técnica:

- Análisis y observación de variables

- Población: terrenos cultivados con diferentes productos de la región (maíz, café, plátano)

- Diseño experimental

- Análisis estadístico de datos

En este proyecto se utilizó la metodología aplicada tecnológica, que procura brindar una solución a una necesidad, en este caso, en el rubro agrícola, 
mediante la implementación de las competencias en las áreas de la ingeniería electrónica y la química, con el objetivo de desarrollar un instrumento innovador de medición de $\mathrm{pH}$ que permitirá al sector agrícola tener una herramienta para los suelos cultivados y así tener un punto de referencia para el beneficio del cultivo y del agricultor.

La metodología de este proyecto se desarrollará en cuatro fases, de acuerdo con los objetivos planteados. A continuación se describen dos de estas [2], entendiendo que las dos últimas están comprendidas en las simulaciones y las pruebas del CIAB Dosquebradas descritas en los resultados:

\section{Fase 1: Recolección de información}

La búsqueda de información teórica documental sobre la variable a trabajar en conjunto con las consultas a expertos (agricultores, agrónomos, ingenieros electrónicos y profesionales químicos) para obtener un marco teórico con las teorías, experimentos y antecedentes que sustenten el proyecto [2].

El pH: Es utilizado en todo el mundo para referenciar la fórmula del potencial de Hidrógeno $(\mathrm{H}+)$, es decir, la cantidad del ion hidrógeno que existe en una solución. Las diferentes sustancias que están a nuestro alrededor poseen un nivel de $\mathrm{pH}$ diferenciado que las caracteriza y las hace útiles o beneficiosas para determinados casos. [2,12]

Fórmula del pH: El químico danés Sören Peter Lauritz Sorensen definió originalmente el pH como el logaritmo negativo de la concentración del ión hidrógeno, es decir [13]:

$p H=-\log \left(H^{+}\right) \rightarrow p H=\log \left(\frac{1}{H^{+}}\right)$

Escala del pH: El estándar internacional propone la escala de 0 a 14, donde 7 es el punto neutro y los extremos se caracterizan por esto:

Inferior a 7: son sustancias ácidas, ceden electrones $\left(\mathrm{H}^{+}\right)$. Sabor agrio, como el jugo de limón y el vinagre, Producen irritación a la piel, causando sensaciones punzantes. En el ámbito biológico son centrales para el metabolismo de las proteínas. Cambian el papel tornasol de azul a rojo. Se disuelven o reaccionan con los metales y neutralizan las bases.

Superior a 7: son sustancias básicas o alcalinas, que captan electrones $\left(H^{+}\right)$. Tienen sabor amargo, son deslizantes al tacto. Las bases fuertes son cáusticas a la piel y las proteínas, tal es el caso de la soda cáustica. Cambian el papel tornasol de rojo a azul y neutralizan los ácidos [2,14]. 


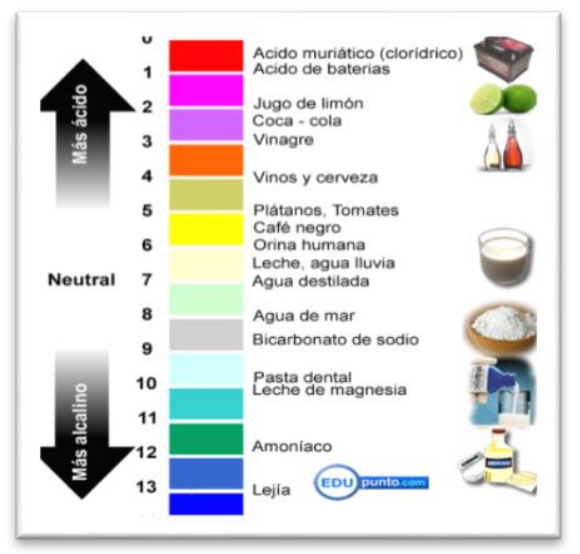

El pH en el suelo: El pH interviene directamente en el desarrollo vegetal, de él depende la disponibilidad y solubilidad de los nutrientes y la materia orgánica encontrados en el suelo. Cada planta necesita elementos en diferentes cantidades y por tanto requieren un rango particular de $\mathrm{pH}$ para optimizar su crecimiento. Cuando disminuye la solubilidad, las plantas no pueden absorber los elementos del suelo; en los otros, el aumento de la solubilidad debida al $\mathrm{pH}$ hará que para determinados elementos sea máxima, llegando al punto de toxicidad [2,5].

\section{Fase 2: Diseño e implementación del dispositivo}

Esta fase consta de dos etapas paralelas para la realización del proyecto, la detección de mejoras y la solución de posibles inconvenientes que puedan surgir en desarrollo del dispositivo:

Etapa 1. Diseño y simulación del circuito electrónico para escalizar la medida tomada por el electrodo y luego visualizarla en un display LCD, realizado con un software para la aplicación.

Etapa 2. Selección e implementación de un electrodo idóneo para la medición de $\mathrm{pH}$ en terreno agrícola, realización del montaje de componentes electrónicos en placa PCB y del display LCD para la toma de las primeras pruebas de funcionamiento [2].

\section{Medición del pH}

Hay varias formas de medir el nivel de $\mathrm{pH}$. Esto depende de la aplicación, del medioambiente (esto es, en un laboratorio, en sólidos, en disolventes, directamente en el suelo, etc.) y de la disponibilidad de recursos [12].

Potenciómetro o pH-metro: Un pH-metro mide la diferencia de voltaje en una disolución, es un voltímetro que junto con los electrodos, al ser sumergidos en una sustancia, generan una corriente eléctrica. Esta corriente eléctrica dependerá de la concentración de iones de hidrógeno [15] que 
presente la solución. La parte más importante del sistema es el electrodo, cuyo potencial depende del $\mathrm{pH}[2,16]$.

Figura 2. pH-metro combinado [15]

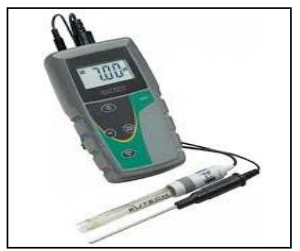

Elección del sensor (o electrodo) de pH: Un sensor es un dispositivo capacitado para medir magnitudes físicas o químicas, llamadas variables de instrumentación, y transformarlas en variables eléctricas. Las variables de instrumentación pueden ser por ejemplo: fuerza, humedad, pH, etc. $[2,13$, 17].

Tabla 1. Comparativa entre los sensores de $\mathrm{pH}[2,17]$

\begin{tabular}{|c|c|c|c|}
\hline & $\begin{array}{c}\text { Omega PHE- } \\
7353\end{array}$ & Fraunhofer & $\begin{array}{l}\text { Ocean } \\
\text { Optics }\end{array}$ \\
\hline Tipo de sensor & Vidrio & ISFET & $\begin{array}{l}\text { Fibra } \\
\text { Óptica }\end{array}$ \\
\hline Rango pH & $0-14$ & $1-12$ & $6.5-12$ \\
\hline $\begin{array}{c}\text { Rango } \\
\text { Temperatura } \\
\left({ }^{\circ} \mathrm{C}\right)\end{array}$ & $0-80$ & $-10-75$ & $0-220$ \\
\hline $\begin{array}{l}\text { Rango Presión } \\
\text { (psi) }\end{array}$ & $0-100$ & $7-145$ & $0-100$ \\
\hline Precisión & $\begin{array}{c}0,1 \% \text { sobre } \\
\text { fondo de } \\
\text { escala }\end{array}$ & 0.02 & $s / d$ \\
\hline $\begin{array}{l}\text { Impedancia de } \\
\text { salida }\end{array}$ & $150 \mathrm{M}$ & $s / d$ & $s / d$ \\
\hline $\begin{array}{c}\text { Tiempo de } \\
\text { respuesta (s) }\end{array}$ & 10 & 60 & $s / d$ \\
\hline $\begin{array}{c}\text { Deriva } \\
\text { (uV/hora) }\end{array}$ & 12 & 80 & $s / d$ \\
\hline $\begin{array}{l}\text { Dimensiones } \\
(\mathrm{mm})\end{array}$ & $152(\mathrm{~L}) \times 22(\mathrm{D})$ & $3.5 * 3.5$ & $108(L) \times 3(D)$ \\
\hline $\begin{array}{l}\text { Compensación } \\
\text { de temperatura }\end{array}$ & $\begin{array}{c}\text { Pt100 } \\
\text { incluida }\end{array}$ & Pt 1000 & NC \\
\hline Mantenimiento & Húmedo & Seco & Seco \\
\hline Costo (U\$D) & 150 & $s / d$ & 990 \\
\hline
\end{tabular}


Debido a los requerimientos del prototipo, se optó por un electrodo de encapsulamiento metálico porque son los más adecuados para mediciones de $\mathrm{pH}$ directas al suelo; este tiene características muy similares al tipo ISFET, con un rango de tensión entre $800 \mathrm{mV}$ a $-800 \mathrm{mV}$ aproximadamente, siendo los valores más próximos a OV los que indican un nivel de $\mathrm{pH} 7$. Las tensiones negativas indican algún grado de acidez, y las tensiones positivas, alcalinidad $[2,14,17]$

- Especificaciones de diseño: Se definieron las siguientes especificaciones para el diseño del prototipo.

- Medición altamente precisa del nivel de pH en el suelo.

- Funcionamiento autónomo, es decir, sin conexión a una toma eléctrica; trabajará con baterías recargables, lo que indica portabilidad.

- Manipulación sencilla por parte del usuario.

- Robustez, debido a que la medición puede desplegarse en ambientes hostiles y terrenos en condiciones adversas.

Descripción general y diseño del instrumento de medición de $\mathrm{pH}$ : $\mathrm{El}$ dispositivo fue constituido por tres módulos:

- Alimentación

- Procesamiento

- Visualización

Básicamente, el primer módulo suple de energía eléctrica continua a todo el circuito; el segundo módulo capta la señal análoga proveniente del sensor o electrodo de $\mathrm{pH}$ y realiza la adecuación de la señal; en tanto que el tercer módulo lleva a cabo su respectiva visualización en tiempo real [2].

El siguiente diagrama ilustra el conducto regular de operación:

Figura 3. Diagrama esquemático del dispositivo [2]

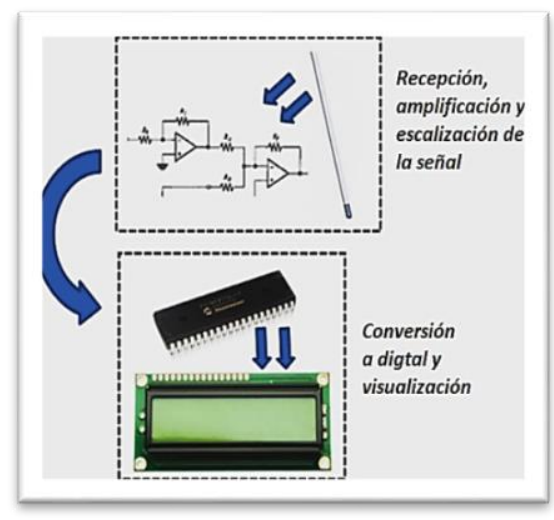

El siguiente es el diagrama de bloques del instrumento: 
Figura 4. Diagrama de bloques del instrumento de medición de pH [2]

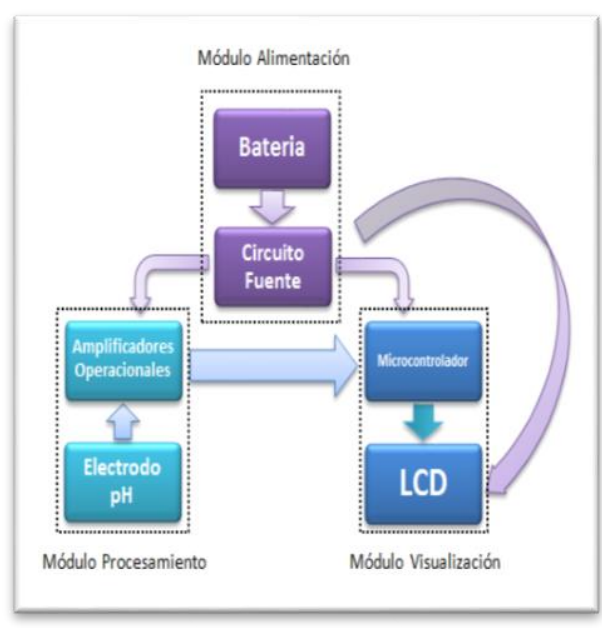

\section{Módulo de alimentación}

Hay varias formas de medir el nivel de $\mathrm{pH}$ según las condiciones de la aplicación, del medioambiente (esto es, en un laboratorio, en sólidos, en disolventes, directamente en el suelo, etc.) y de la disponibilidad de recursos; con un voltaje de carga de 9 o 12 voltios [2]. Esta fuente entregará al resto del circuito dos tensiones, una de 5 voltios positivos y la otra de 5 voltios negativos $[15,18]$.

Figura 5. Módulo alimentación [2]

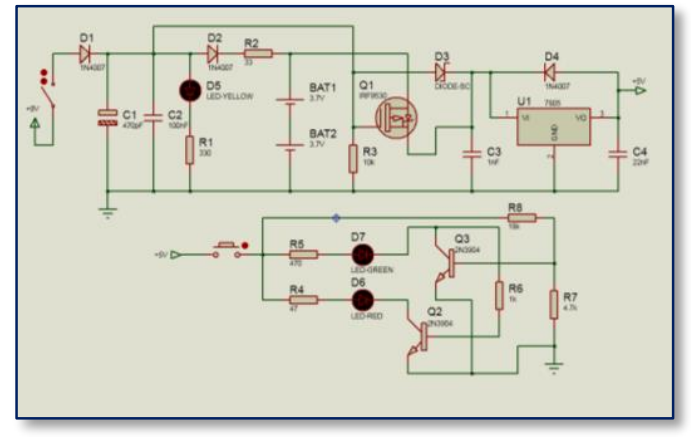

Este tipo de circuito también es conocido como fuente de voltaje con carga compartida, ello significa que mientras el dispositivo recarga la batería puede seguir suministrando voltaje a los demás circuitos.

La tensión recibida del cargador, que puede ser de 12 o 9 voltios, llega al regulador de voltaje LM317, el cual baja la tensión a unos 8.5 voltios, apropiados para recargar la batería de litio y una corriente de 2 amperios. Los capacitores cerámicos al igual que los electrolíticos cumplen con la función de filtrar y estabilizar la tensión.

El otro regulador de voltaje es un 7805. Este también recibe la tensión del cargador para regularlo a 5 voltios. Con este voltaje trabajará la mayoría de componentes del circuito, por ejemplo: el microcontrolador, la pantalla LCD, y parte de los amplificadores operacionales [2]. 
Los amplificadores operacionales del módulo de procesamiento trabajan con voltaje positivo y voltaje negativo; por tal motivo, el circuito de alimentación cuenta con un convertidor de voltaje a negativo, se trata del ICL7660. Este integrado realiza la conversión de la tensión de alimentación de positivo a negativo para un rango de entrada de $1.5 \mathrm{~V}$ a $12 \mathrm{~V}$, lo que resulta en tensiones de salida complementarias de $-1.5 \mathrm{~V}$ a $-12 \mathrm{~V}$. Solo se necesitan 2 condensadores externos para cumplir funciones de depósito de la bomba y de carga $[2,16]$.

\section{Módulo de procesamiento}

En este circuito se realiza la toma de la lectura de $\mathrm{pH}$ hecha por el electrodo, el cual, como se ha tratado anteriormente, genera una diferencia de potencial cerca de los 400 mili-voltios. Gracias a la alta impedancia de este circuito, el voltaje es amplificado y posteriormente se ajusta para convertirlo en una escala de 0 a 5 voltios, con lo cual podrá trabajar el microcontrolador en el próximo módulo. El uso de potenciómetros permite realizar la calibración del circuito y, por lo tanto, del instrumento $[2,17,18,19]$.

Figura 6. Módulo de procesamiento [2]

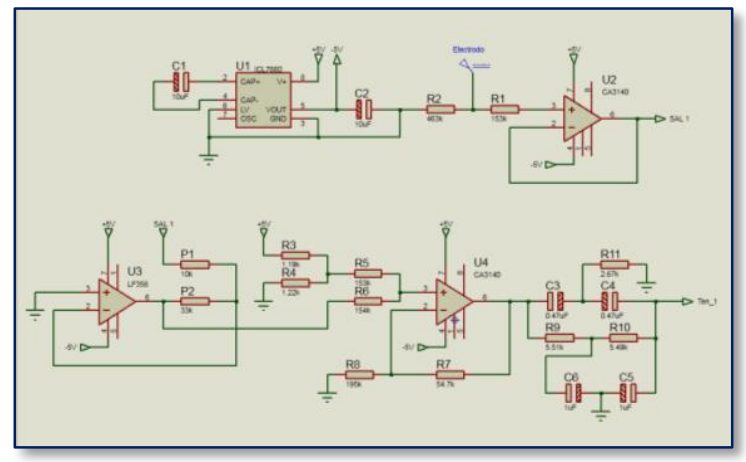

\section{Módulo de visualización}

En este punto encontramos el microcontrolador PIC16F877A, que trabaja con una frecuencia de $4 \mathrm{MHz}$. La tensión escalizada de 0 a 5 voltios llega al pin 2. Esta es la señal procesada por los amplificadores operacionales del módulo visto anteriormente y viene acomodada en valores que el PIC puede trabajar. El programa compilado en el PIC traduce la escala de tensión de la señal de entrada en la escala de $\mathrm{pH}$, es decir, de 0 a 14. Por último, envía los datos al controlador interno de la pantalla LCD $16 \times 2$ y así se visualiza el resultado de la medición de $\mathrm{pH}[18,19]$. 
Figura 7. Módulo visualización [2]

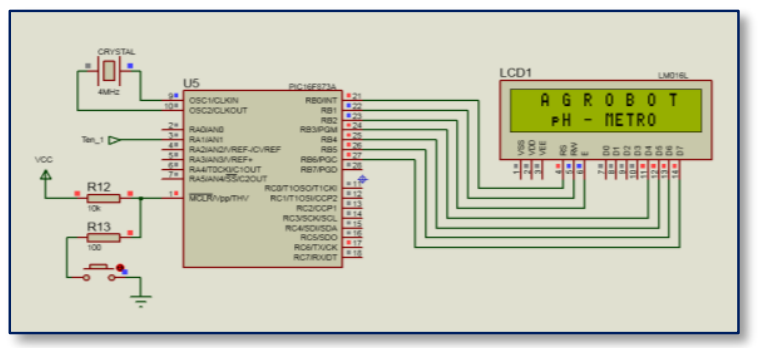

\section{Diseño de algoritmo y software}

El programa que lleva el microcontrolador fue realizado en lenguaje $C$, de fácil comprensión. Este configura el PIC para recibir la tensión escalizada de 0 a 5 voltios por el pin 3, lo cual es en sí una señal ya procesada por los amplificadores operacionales del módulo visto anteriormente. Ajustada en valores que el PIC puede trabajar, el programa traduce la escala de voltaje en la escala de nivel de $\mathrm{pH}$, es decir, de 0 a 14. Por último, envía los datos al controlador interno de la pantalla LCD $16 \times 2$ y así se puede visualizar el resultado de la medición de $\mathrm{pH}[2,19]$.

Tabla 2. Conversión y escalización de voltajes [2]

\begin{tabular}{|c|c|c|}
\hline Tensión del electrodo (milivoltios) & $\begin{array}{c}\text { Tensión al PIC } \\
\text { (voltios) }\end{array}$ & $\begin{array}{c}\text { Equivalencia } \\
\text { en } \mathrm{pH} \\
\text { (nivel) }\end{array}$ \\
\hline-400 a -468 & 2,47 a 2,62 & $\begin{array}{c}\text { Entre } 3 y \\
3,99\end{array}$ \\
\hline-470 a -490 & 2,63 a 2,67 & $\begin{array}{c}\text { Entre } 4 \mathrm{y} \\
4,99\end{array}$ \\
\hline-280 a -340 & 2,21 a 2,35 & $\begin{array}{c}\text { Entre } 5 \text { y } \\
5,99\end{array}$ \\
\hline-80 a -270 & 1,87 a 2,2 & $\begin{array}{c}\text { Entre } 6 \mathrm{y} \\
6,99\end{array}$ \\
\hline-22 a -99 & 1,68 a 1,84 & $\begin{array}{c}\text { Entre } 7 \mathrm{y} \\
7,99\end{array}$ \\
\hline 10 a 210 & 1,19 a 1,61 & $\begin{array}{c}\text { Entre } 8 y \\
9,99\end{array}$ \\
\hline
\end{tabular}

\section{Resultados y discusión}

\section{Simulación de medición de una sustancia ácida}

La primera simulación se realiza a lo que puede ser una sustancia con un valor de pH cercano a 3, es decir, ácidos como el zumo de limón.

- Tensión generada por el electrodo: $-450 \mathrm{mV}$

- Tensión al PIC: 2,58V

- $\quad$ Nivel de $\mathrm{pH}: 3,77$ 
Figura 8. Simulación 1 módulo procesamiento [2]

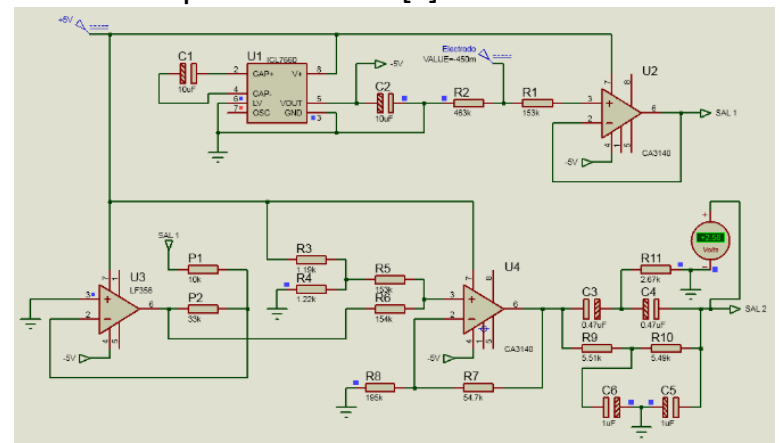

Figura 9. Simulación 1 módulo visualización [2]

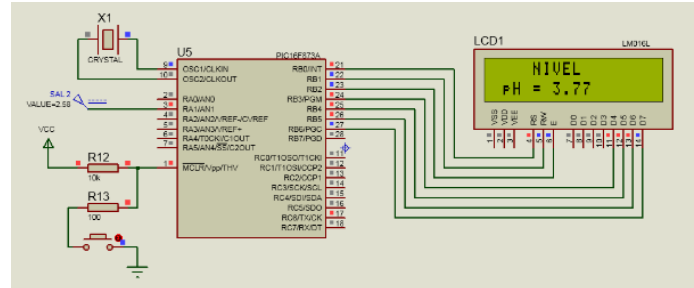

\section{Simulación de medición de una sustancia neutra}

La simulación se realiza a lo que puede ser una sustancia con un valor de $\mathrm{pH}$ cercano a 7, es decir, agua pura.

- Tensión generada por el electrodo: $-50 \mathrm{mV}$

- Tensión al PIC: $1,74 \mathrm{~V}$

- Nivel de $\mathrm{pH}: 7,33$

Figura 10. Simulación 2 módulo procesamiento [2]

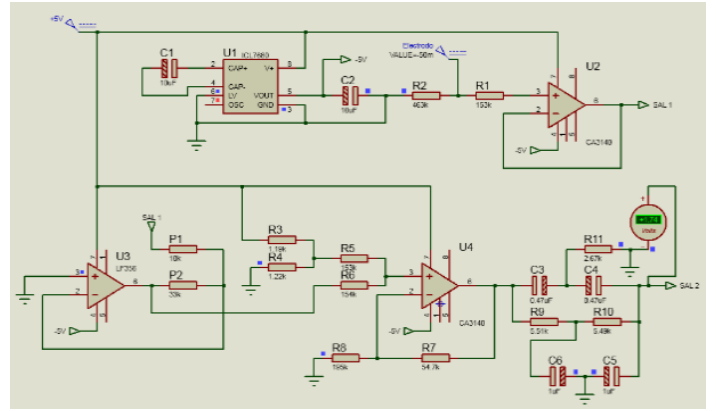

Figura 11. Simulación 2 módulo visualización [2]

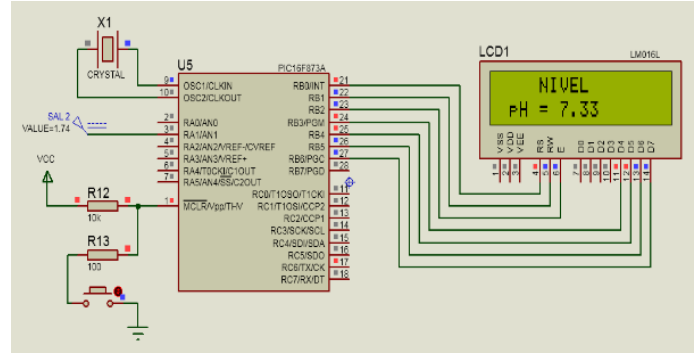


Simulación de medición de una sustancia básica o alcalina La simulación se realiza a lo que puede ser una sustancia con un valor de $\mathrm{pH}$ cercano a 7, es decir, agua pura.

- Tensión generada por el electrodo: $210 \mathrm{mV}$

- Tensión al PIC: 1,19V

- Nivel de pH: 8,90 (Figura 13)

Figura 12. Simulación 3 módulo procesamiento [2]

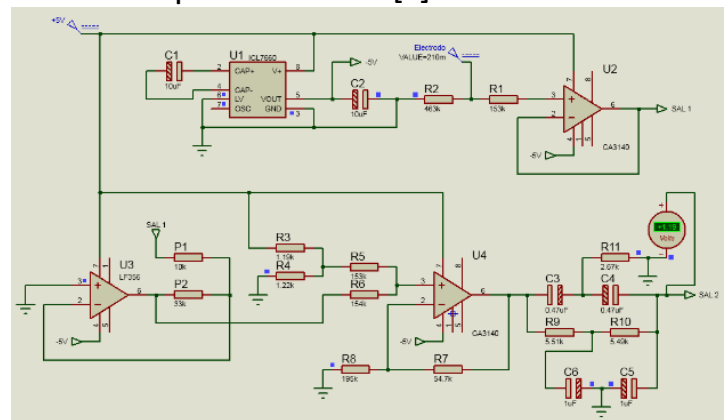

Figura 13. Simulación 3 módulo visualización [2]

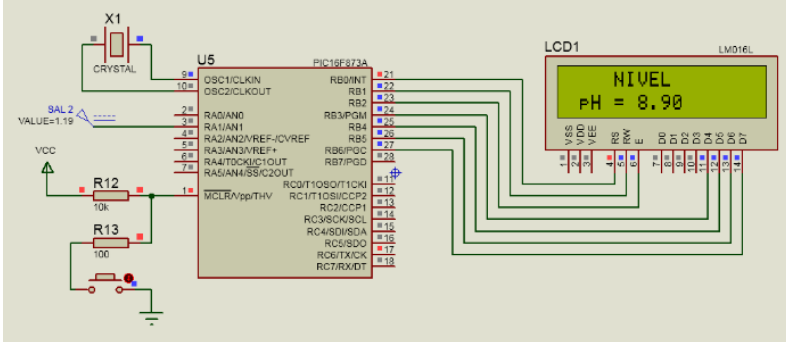

\section{Pruebas en terreno}

Tabla 3. Muestra 1 Fecha 04 Abril 2017 (Rovira-Tolima) [2]

\begin{tabular}{|c|c|c|}
\hline Tiempo & $\begin{array}{c}\text { Prototipo 10:30 } \\
\text { am }\end{array}$ & \begin{tabular}{c} 
Ph-Metro Hanna 10:40 am \\
\hline Inicial
\end{tabular} 7.11 \\
\hline Min 1 & 7.6 & 5.78 \\
\hline Min 2 & 7.56 & 5.68 \\
\hline Min 3 & 7.67 & 5.65 \\
\hline
\end{tabular}

De acuerdo con la Muestra No 1, correspondiente a la pruebas de $\mathrm{pH}$ en el Municipio de Rovira, esta primera muestra del prototipo está 1,33 unidades por debajo del resultado óptimo, lo que correspondería a un $18,70 \%$ de variación. La segunda muestra d

el prototipo est 1,92 unidades por debajo del resultado óptimo, lo que correspondería a un 25,26 \% de variación. En tanto que la tercera 'se halla 
1,91unidades por debajo del resultado óptimo, lo que correspondería a un $25,26 \%$ de variación. La cuarta muestra, por su parte, se encuentra 2,06 unidades por debajo del resultado óptimo, lo que correspondería a un 26,85 $\%$ de variación.

Es de resaltar que los cuatro resultados arrojados con el instrumento diseñado están alejados del resultado óptimo del prototipo, lo que nos permite concluir que el instrumento utilizado en esta medición debe ser revisado o calibrado para que cumpla con los parámetros óptimos de diseño.

Si tenemos en cuenta el resultado promedio, podemos concluir, además, hay una variación en relación con el resultado del prototipo en un promedio de $-1,805$ unidades, lo que significa que está por debajo del promedio en un $24,11 \%$ de variación.

Figura 14. Medición sobre zumo de limón, pH aproximado 3

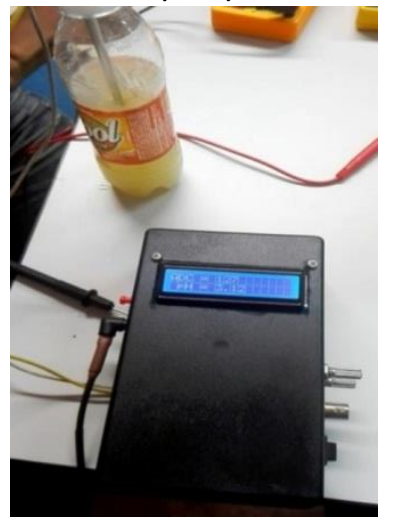

Tabla 4. Muestra 4 Fecha 09 Junio 2017 (Armero)

\begin{tabular}{|c|c|c|}
\hline Tiempo & Prototipo 11:30 am & Ph-Metro Hanna \\
\hline Inicial & 8.0 & 6.92 \\
\hline Min 1 & 7.22 & 6.60 \\
\hline Min 2 & 7.11 & 6.66 \\
\hline Min 3 & 7.11 & 6.62 \\
\hline
\end{tabular}

\section{Análisis de las pruebas en la Finca "La Joya":}

En las Pruebas de campo realizadas y de acuerdo con los datos recogidos, el Instrumento de Medición nos da unos valores muy estables, las cuales se están acercando visiblemente a nuestro objetivo al diseñar los sensores para que sea comparable con $\mathrm{pH}$-metro de laboratorio o profesionales.

Según la Muestra No. 4, correspondiente a la pruebas de pH en el Municipio de Armero-Guayabal, la primera muestra está 1,08 unidades por debajo del resultado óptimo, lo que correspondería a un 13,5 \% de variación. La segunda muestra se halla 0,62 unidades por debajo del resultado óptimo, 
lo que correspondería a un $8,58 \%$ de variación: La tercera muestra está 0,45 unidades por debajo del resultado óptimo, lo que correspondería a un $6,32 \%$ de variación. Por último, la cuarta muestra está 0,49 unidades por debajo del resultado óptimo, lo que correspondería a un 6,89 \% de variación

Los cuatro resultados anteriores están muy cerca del resultado óptimo del prototipo, lo que nos permite concluir que en este caso el instrumento de medición utilizado cumple con los parámetros óptimos de diseño para la medición del pH.

Figura 15. Datos muestra N 4 Armero

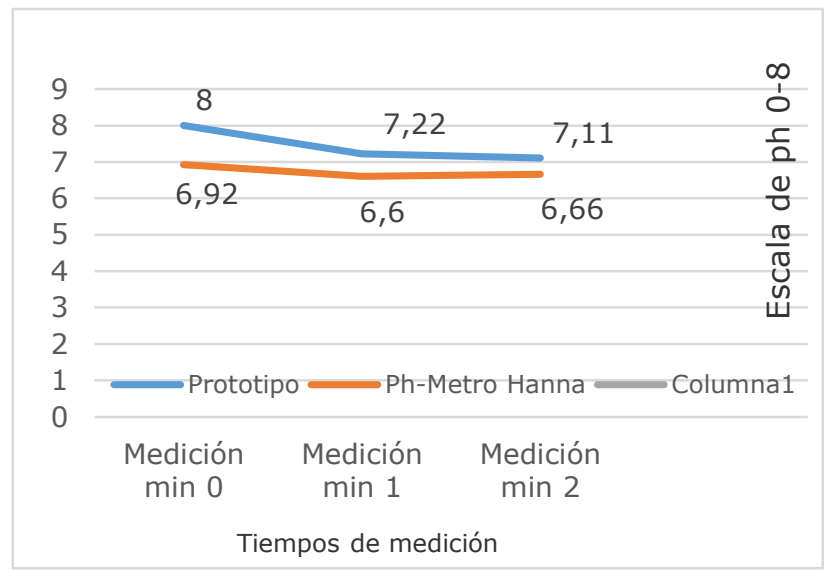

Si tenemos en cuenta el resultado promedio, podemos concluir que el resultado con el instrumento presenta una variación en relación con el resultado del prototipo en un promedio de $-0,66$ unidades. Esto significa que se halla por debajo del promedio en un $8,96 \%$ de variación

La cuarta prueba de medición con sensor de $\mathrm{pH}$ prototipo más $\mathrm{pH}$ metro Hanna en el Ciap de Pereira evidencia que las mediciones de $\mathrm{pH}$ realizadas hasta el momento se han hecho a partir de la comparación de los resultados contra un pH metro de laboratorio y de toma de muestra húmeda, preparada en laboratorio, técnica muy diferente a la del pH metro diseñado por este proceso de investigación. Por eso y debido a las diferencias marcadas de resultados, se buscaba un tercer tipo de comparación con el tratamiento necesario para llegar a una correlación entre un sensor de bulbo seco y uno de bulbo húmedo.

Pruebas finales realizadas en el Centro de Investigación de Agricultura y Biotecnología-CIAB del municipio de Dos Quebradas.

\section{Metodología}

Se realizaron las mediciones comparativas entre el sensor HANNA de laboratorio, el sensor Consort inalámbrico del CIAB (Centro de la investigación de Agricultura y Biotecnología) en Pereira y los resultados 
otorgados por el instrumento de medición diseñado por los miembros del semillero SIART, Estas mediciones se realizaron en el municipio de Dosquebradas cerca de Pereira, donde se encuentra el CIAB de la universidad UNAD, entre las 9am y las $6 \mathrm{pm}$ del día viernes 04 de agosto y las $8 \mathrm{am}$ a $12 \mathrm{~m}$ del sábado 05 de agosto.

Antes de realizar las pruebas se llevó a cabo un recorrido por las instalaciones del CIAB para conocer sus equipos y el área donde se realizarían las pruebas. Se nos explicó el funcionamiento del sistema AUTOGRONOM, el cual controla el pH del invernadero por medio de una solución de nutrientes que estabiliza o altera el valor del $\mathrm{pH}$ del terreno, según lo necesitado o el experimento que esté llevando a cabo el investigador.

Así mismo, se nos explicó los funcionamientos de las bombas y válvulas que realizan las funciones anteriormente mencionadas para la alteración del terreno en el invernadero.

Figura 16. Sistema de control Auto-gronom - CIAB

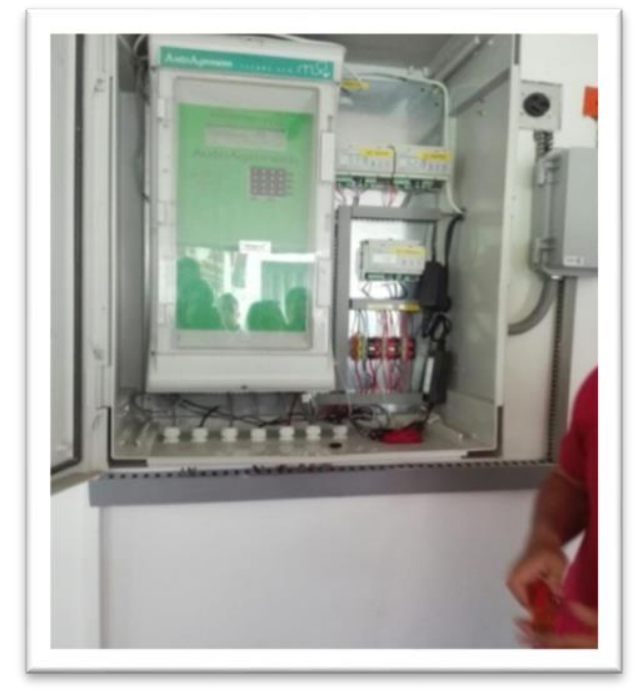

Se seleccionan los equipos a trabajar, en este caso particular, el sensor Inalámbrico del CIAB para compararlo contra el sensor prototipo y el $\mathrm{pH}$ metro HANNA con los mismos elementos.

Se tomaron prestadas tres probetas de la sala anexa de la sala de control del sistema AUTO-GRONOM para realizar las pruebas en los elementos.

Se tomaron las mediciones de tres minutos, registrando los resultados y anotando el comportamiento de los $\mathrm{pH}$-metros.

Se procedió a limpiar los pH-metro de residuos con agua destilada y a ingresarles en sus respectivas capuchas protectoras. Luego se limpiaron con la solución de almacenamiento de $\mathrm{Ph}$ 70/300 para el mantenimiento óptimo del equipo de bulbo, así mismo, se guardó el electrodo del prototipo con medidas de protección. 
Tabla 5. Mediciones en instante inicial

\begin{tabular}{|c|c|c|c|}
\hline $\begin{array}{c}\text { Tiempo } \\
\text { Inicial }\end{array}$ & Prototipo & Ph-Metro Hanna & Ph-Metro Consort \\
\hline muestra 1 & $\mathbf{7 , 0 0}$ & $\mathbf{6 , 5 0}$ & $\mathbf{6 , 8 0}$ \\
\hline muestra 2 & $\mathbf{6 , 5 0}$ & $\mathbf{6 , 8 0}$ & $\mathbf{6 , 3 4}$ \\
\hline muestra 3 & $\mathbf{5 , 2 8}$ & $\mathbf{3 , 1 2}$ & $\mathbf{2 , 7 0}$ \\
\hline muestra 4 & $\mathbf{7 , 0 0}$ & $\mathbf{6 , 5 0}$ & $\mathbf{6 , 8 0}$ \\
\hline muestra 5 & $\mathbf{8 , 0 4}$ & $\mathbf{6 , 5 0}$ & $\mathbf{6 , 8 0}$ \\
\hline muestra 6 & $\mathbf{8 , 0 4}$ & $\mathbf{7 , 2 5}$ & $\mathbf{7 , 5 0}$ \\
\hline muestra 7 & $\mathbf{6 , 9 6}$ & $\mathbf{6 , 6 2}$ & $\mathbf{6 , 7 7}$ \\
\hline muestra 8 & $\mathbf{6 , 7 6}$ & $\mathbf{6 , 8 2}$ & $\mathbf{6 , 4 0}$ \\
\hline muestra 9 & $\mathbf{4 , 5 1}$ & $\mathbf{3 , 0 9}$ & $\mathbf{2 , 8 0}$ \\
\hline muestra 10 & $\mathbf{7 , 1 4}$ & $\mathbf{5 , 5 4}$ & $\mathbf{5 , 2 0}$ \\
\hline muestra 11 & $\mathbf{7 , 0 4}$ & $\mathbf{7 , 2 7}$ & $\mathbf{6 , 8 0}$ \\
\hline muestra 12 & $\mathbf{7 , 0 0}$ & $\mathbf{6 , 5 0}$ & $\mathbf{6 , 8 0}$ \\
\hline
\end{tabular}

Figura 17. Mediciones momento inicial

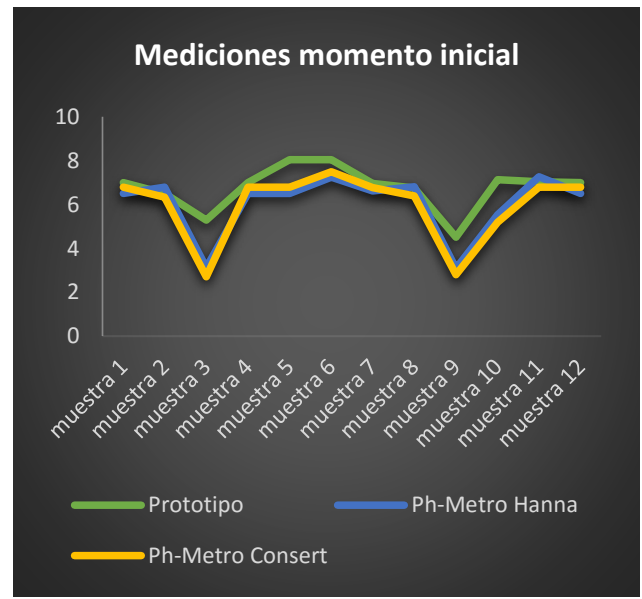

El análisis de los datos del momento inicial permite concluir que:

En el momento que se mide un valor de $\mathrm{pH}$ por debajo del rango de trabajo del prototipo, la medida dista mucho de acercarse a lo esperado, obteniendo una diferencia promedio del $35.5 \%$ respecto a los $\mathrm{pH}$-metros de referencia.

Al medir valores de $\mathrm{pH}$ en el límite justo del rango de trabajo (4.01), se obtiene una diferencia promedio del $7.14 \%$ respecto a los $\mathrm{pH}$-metros de referencia. 
Al medir valores de $\mathrm{pH}$ dentro del rango de trabajo, sin acercarse mucho a los límites (entre 5 y 7.5), se obtiene una diferencia promedio de $7.11 \%$ respecto a los $\mathrm{pH}$-metros de referencia.

Tabla 6. Mediciones minuto 1

\begin{tabular}{|c|c|c|c|}
\hline $\begin{array}{c}\text { Tiempo } \\
\text { Inicial }\end{array}$ & Prototipo & Ph-Metro Hanna & Ph-Metro Consort \\
\hline muestra 1 & $\mathbf{7 , 1 2}$ & $\mathbf{7 , 0 2}$ & $\mathbf{7 , 0 1}$ \\
\hline muestra 2 & $\mathbf{6 , 8 2}$ & $\mathbf{6 , 7 5}$ & $\mathbf{5 , 7 8}$ \\
\hline muestra 3 & $\mathbf{4 , 7 5}$ & $\mathbf{3 , 1 0}$ & $\mathbf{2 , 7 5}$ \\
\hline muestra 4 & $\mathbf{5 , 5 0}$ & $\mathbf{5 , 4 5}$ & $\mathbf{6 , 1 0}$ \\
\hline muestra 5 & $\mathbf{7 , 3 7}$ & $\mathbf{7 , 1 5}$ & $\mathbf{7 , 0 0}$ \\
\hline muestra 6 & $\mathbf{7 , 1 9}$ & $\mathbf{7 , 0 2}$ & $\mathbf{7 , 0 1}$ \\
\hline muestra 7 & $\mathbf{7 , 0 0}$ & $\mathbf{6 , 9 0}$ & $\mathbf{7 , 0 1}$ \\
\hline muestra 8 & $\mathbf{6 , 8 1}$ & $\mathbf{6 , 7 8}$ & $\mathbf{6 , 5 5}$ \\
\hline muestra 9 & $\mathbf{4 , 3 3}$ & $\mathbf{3 , 0 2}$ & $\mathbf{4 , 8 0}$ \\
\hline muestra 10 & $\mathbf{5 , 5 2}$ & $\mathbf{4 , 3 9}$ & $\mathbf{7 , 0 0}$ \\
\hline muestra 11 & $\mathbf{6 , 9 0}$ & $\mathbf{7 , 1 5}$ & $\mathbf{6 , 3 4}$ \\
\hline muestra 12 & $\mathbf{6 , 5 0}$ & $\mathbf{6 , 8 0}$ & \\
\hline
\end{tabular}

Figura 18. Mediciones minuto 1

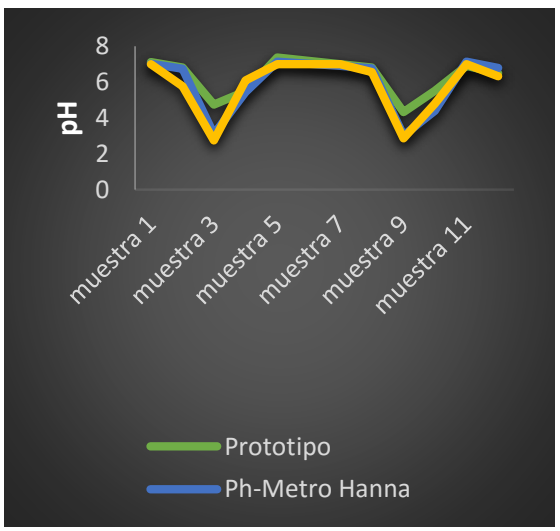

El análisis de los datos al minuto 1 de tener los sensores en proceso de medición permite observar que:

Al medir un valor de $\mathrm{pH}$ por debajo del rango de trabajo del prototipo, la medida presenta una diferencia promedio del $32.5 \%$ respecto a los $\mathrm{pH}$ metros de referencia. En este momento la medida promedio del prototipo es $13.5 \%$ mayor al límite del rango de trabajo.

Al medir valores de $\mathrm{pH}$ en el límite justo del rango de trabajo (4.01), se obtiene una diferencia promedio del $2.76 \%$ respecto a los $\mathrm{pH}$-metros de referencia. 
Al medir valores de $\mathrm{pH}$ dentro del rango de trabajo, sin acercarse mucho a los límites (entre 5 y 7.5), se obtiene una diferencia promedio de $1.89 \%$ respecto a los $\mathrm{pH}$-metros de referencia.

Tabla 7. Mediciones minuto 2

\begin{tabular}{|c|c|c|c|}
\hline Tiempo & Prototipo & Ph-Metro Hanna & Ph-Metro Consort \\
\hline muestra 1 & 7,10 & 7,01 & - \\
\hline muestra 2 & 6,87 & 6,72 & 5,79 \\
\hline muestra 3 & 4,23 & 3,08 & 2,79 \\
\hline muestra 4 & 4,45 & 4,33 & 5,01 \\
\hline muestra 5 & 6,33 & 7,01 & - \\
\hline muestra 6 & 7,01 & 7,01 & - \\
\hline muestra 7 & 7,01 & 7,01 & 7,01 \\
\hline muestra 8 & 6,82 & 6,74 & 6,69 \\
\hline muestra 9 & 4,17 & 2,98 & 2,90 \\
\hline muestra 10 & 4,35 & 4,23 & 4,41 \\
\hline muestra 11 & 6,83 & 7,01 & 6,84 \\
\hline muestra 12 & 6,72 & 6,82 & 6,44 \\
\hline
\end{tabular}

Figura 19. Mediciones minuto 2

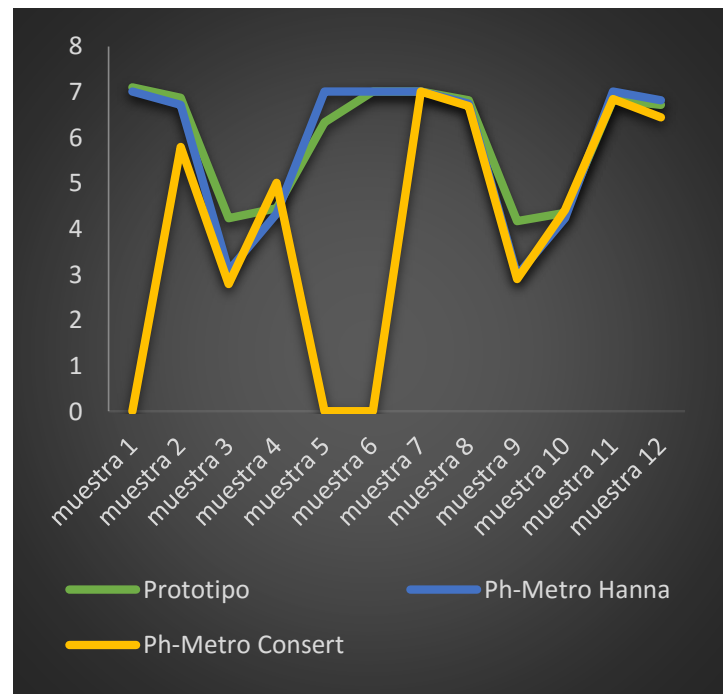

El análisis de los datos al minuto 2 permite observar que:

Al medir un valor de $\mathrm{pH}$ por debajo del rango de trabajo del prototipo la medida presenta una diferencia promedio del $27.8 \%$ respecto a los $\mathrm{pH}$ metros de referencia; se resalta que en este momento la medida del 
prototipo tenía una diferencia del $7.7 \%$ respecto al límite del rango de trabajo.

Al medir valores de $\mathrm{pH}$ en el límite justo del rango de trabajo (4.01), se obtiene una diferencia promedio del $2.09 \%$ respecto a los $\mathrm{pH}$-metros de referencia.

Al medir valores de $\mathrm{pH}$ dentro del rango de trabajo, sin acercarse mucho a los límites (entre 5 y 7.5), se obtiene una diferencia promedio de $2.5 \%$ respecto a los $\mathrm{pH}$-metros de referencia.

\section{Beneficios para la comunidad e impacto social}

El instrumento de medición de pH es una herramienta que permite tener el valor de medida real, especificando el nivel de acidez o alcalinidad del suelo; esta beneficia directamente al agricultor ya que al tener la información veraz junto a la aplicación del proceso adecuado según el resultado dado por la medición de $\mathrm{pH}$, obtendrá cosechas de alta calidad [2, 20].

El potencial uso de este versátil instrumento de medición corresponde a cada una de las personas que trabajan la agricultura, como los agrónomos, ingenieros agro-forestales, campesinos, amantes de la jardinería o sencillamente la persona que desee conocer el valor del $\mathrm{pH}$ de la tierra dispuesta para cultivo.

Otro de los beneficios que brinda este proyecto a la comunidad en general y muy especialmente a los agricultores o amantes de las plantas es que el bajo costo del procedimiento [2].

\section{Impacto ambiental del proyecto}

De acuerdo con la norma técnica colombiana NTC 5264 [21,22, 23,24], los suelos para cultivo deben tener un rango de $\mathrm{pH}$ determinado, por ello, el desconocimiento de los niveles de $\mathrm{pH}$ en los suelos de cultivos es un problema considerable. El uso inadecuado de fertilizantes, químicos y riego dañan la tierra haciendo que a mediano y largo plazo pierda su fertilidad; de igual manera, es un problema de salud nutricional, pues, los productos del campo con índices desproporcionados de $\mathrm{pH}$ constituyen una amenaza directa para la población $[25,26,27,28,29,30,31,32,33,34]$.

Este proyecto permite entregar información confiable y precisa sobre el nivel de $\mathrm{pH}$ en los suelos de los cultivos al agrónomo. Se presume que este implementará los tratamientos correctivos cuando los niveles de pH estén fuera del estándar saludable, en consecuencia, habrá productos agrícolas de mayor calidad y terrenos bien tratados y aprovechados [2].

\section{Conclusiones}

Según todo lo mencionado, el prototipo de $\mathrm{pH}$-metro desarrollado se acerca a las expectativas de diseño que se plantearon en un principio, obteniendo 
una disminución notoria en los costos de implementación del prototipo (alrededor del 50\%) respecto a los $\mathrm{pH}$-metros usados para realizar las pruebas, lo que garantiza confiabilidad en la medición.

El error de la medición en el prototipo es inferior al 3\% luego del minuto 1 de iniciar la medición. Este valor se obtuvo en las pruebas finales realizadas frente a dos sensores de laboratorio. El prototipo presenta una medición confiable entre los minutos 1 y 3, dejarlo más tiempo puede ocasionar saturaciones en la sonda, que se traducen en lecturas erróneas del valor medido.

El prototipo implementado permite medir de manera confiable en un rango de trabajo determinado por valores de $\mathrm{pH}$ entre 4 y 8 ; esta zona de trabajo se justifica ya que que los valores óptimos de $\mathrm{pH}$ en el suelo para la siembra de cultivos se encuentran entre 5 y 7 . Al realizar mediciones fuera de estos rangos, el prototipo demora entre 2 y 3 minutos en llegar hasta el límite inferior o superior de la zona de trabajo establecida.

También se confirmó que la medición de esta variable se ve afectada directamente por otras como la temperatura y la humedad atmosférica. En tal sentido, se aclara que la medición de $\mathrm{pH}$ obtenida es una referencia para el agricultor, mas no se puede asegurar que esta medida no varíe.

La medición de $\mathrm{pH}$ en el suelo, concretamente en tierras dispuestas para cultivos, podrá ser tomada de manera confiable con el prototipo implementado mediante una medición rápida y su procedimiento toma entre 3 y 5 minutos. Esta medida permitirá que se tomen decisiones sobre el manejo del suelo, de manera pronta, permitiendo al productor ahorrar recursos en agroinsumos y lograr más precisión al aplicarlo.

\section{Referencias bibliográficas}

1. Fundacion CANNA, Disponible en: «http://www.canna.es

2. Y. E. Valderrama Lugo, "Diseño e implementación de un instrumento electrónico de medida de pH para terreno agrícola", Repositorio Institucional UNAD, Ibagué, 2017.

3. LeroyMerlin, "LeroyMerlin" [En línea], 2017. Disponible en: https://comunidad.leroymerlin.es/t5/Bricopedia-

Jardiner\%C3\%ADa/Cu\%C3\%A1l-es-la-importancia-del-pH-para-laagricultura/ta-p/157060

4. Agroes,

[En línea].

Disponible

en: http://www.agroes.es/agricultura/el-suelo/148-ph-del-sueloagricultura.

5. Madridmasd. [En línea], 2007. Disponible en: http://www.madrimasd.org/blogs/universo/2007/05/09/65262 
6. Lesco Quimicos, [En línea], 2017. Disponible en: http://lescoquimicos.com/phmetro.htm

7. Infoagro, [En línea], $2017 . \quad$ Disponible en: http://www.infoagro.com/instrumentos_medida/medidor.asp?id=5 412\#precios_modelos.

8. D. Castro Malagon, [En línea], 2003. Disponible en: http://www.docentes.unal.edu.co/glvasque/docs/SUELOS\%20DE\%2 OCOLOMBIA.pdf

9. F. Millan, J. Prato, Y. La Cruz y A. Sánchez, "Estudio metodológico sobre la medición de $\mathrm{pH}$ y conductividad eléctrica en muestras de compost", Revista Colombiana de Química, vol. 47, pp. 21-27, 2018.

10. hydrocultura, [En línea], 2016 Available: https://hydrocultura.com/blogs/noticias-agriculturamexico/importancia-del-ph-en-la-agricultura

11. L. Curto, M. Covi, M. Gassmann, M. Cambareri y A. Maggiora, "Calibración de datos observados de contenido de agua en el suelo con sensores capacitivos", Meteorologica, vol. 41, pp. 49-63, 2016.

12. A. Beretta, D. Bassahun, R. Muselli y D. Torres, "Medición de pH del suelo con papel reactivo", Agrociencia Uruguay, vol. 19, pp. 68-74, 2015.

13. J. Somoza, J. Eirez, S. Pavoni y E. Rodriguez, "Construcción y caracterización de electrodos de Vidrio/ITO/PANI para la medición de $\mathrm{pH} "$, Ingeniería Electrónica, Automática y Comunicaciones, vol. 35, pp. 33-38, 2014.

14. A. Salazar y B. Soriano, "Convertidor de $\mathrm{pH}$ a tensión con compensación automática de temperatura", Tecnología Química, vol. 18, pp. 81-86, 1998.

15. S. Dussan, O. Vanegas , A. Chavarro y J. Molina, "Diseño e implementación de un prototipo electrónico para monitoreo de parámetros físico-químicos en cultivo de tilapia a través de una aplicación móvil", Informador Técnico, vol. 80, pp. 49-60, 2016.

16. J. Gonzalez de la Rosa, Circuitos electrónicos aplicados con amplificadores operacionales: teoría y problemas. España: Servicio de Publicaciones de la Universidad de Cádiz, 2013.

17. L. Diaz y A. Lopez, "Metodología de diseño electrónico aplicado en circuito detector de agua", Redes de Ingeniería, vol. 9, 2018. 
18. J. Mayuza, I. Mariscal y E. Quintero, "Sistema para el monitoreo remoto de la temperatura en transformadores de distribución", Scientia et Technica, vol. 20, pp. 315-322, 2015.

19. I. Dogan, Programación de microcontroladores PIC, Castilla: Marcombo, 2008.

20. M. Delgado, M. Vanegas y G. Delgado, "Metrología Química I: Calibración de un pHmetro y Control de Calidad", Revista Científica de la UNAN-León, vol. 1, n,1, pp. 14-20, 2007.

21. P. Zapata, G. Fuentes, J. Adame y E. Aréchiga, "Efecto del pH y de la Fuente de Carbono Sobre el Crecimiento Vegetativo de Ustilago cynodontis (Pass.) Henn. en medio de cultivo sólido y líquido", Revista Mexicana de Fitopatología, vol. 28, pp. 159-161, 2010.

22. Instituto Colombiano de Normas Técnicas y Certificación, Norma Técnica Colombiana NTC 5264: Calidad de suelo. Determinación del pH., Bogotá: ICONTEC, 2014.

23. W. Lozano Rivas, Suelos : Guía de prácticas simplificadas en campo y laboratorio, Bogotá: eBook Collection (EBSCOhost), 2016.

24. Traxco.es [En línea], $2013 . \quad$ Available: https://www.traxco.es/blog/labores-del-campo/influencia-del-phen-los-sistemas-de-cultivo.

25. Hanna Colombia, [En línea], $2014 . \quad$ Available: http://www.hannacolombia.com/blog/item/457-fotometrosmultiparametricos-fertilizacion-pesticidas-suelosfitosanitarias?tmpl=component\&print=1.

26. J. Navarro., L. Perez., J. Molina., N. Blanco., M. Amador., E. Vasquez., C. Alvarino. Factores intralaborales asociados al síndrome del túnel del carpo en Instrumentadores quirúrgicos en una IPS de Barranquilla. En N. A. (Corporación U. L. León Castro (Ed.), Seguridad y salud en el trabajo: Análisis de las empresas de Barranquilla (Primera, pp. 10-30). Barranquilla: Corporación Universitaria Latinoamericana., 2018.

27. J. Navarro., L. Perez., J. Molina., M. Amador., y E. Vasquez. Esquema de vacunación requerido para el desarrollo de las practicas formativas de los estudiantes de instrumentación quirúrgica. En $\mathrm{N}$. A. (Corporación U. L. León Castro (Ed.), Seguridad y salud en el trabajo: Análisis de las empresas de Barranquilla (Primera, pp. 111130). Barranquilla: Corporación Universitaria Latinoamericana., 2018. 
28. L. Carvajal, [En línea], 2013. Available: http://www.lizardocarvajal.com/investigacionaplicada//docs/SUELOS\%20DE\%20COLOMBIA.pdf.

29. Importancia del pH. [En línea], 2016. Available: https://www.importancia.org/ph.php.

30. instrumentos de laboratorio, ph-metro [En línea], 2014. Available: http://instrumentosdelaboratorio.org/phmetro.

31. J. Oreggioni, "Metodos de medida del ph para estudios invasivos prolongados", [En línea], 2006.

32. R. Areny, Instrumentos Electrónicos Básicos, Marcombo, 2006.

33. F. Valdes y R. Areny, Microcontroladores: fundamentos y aplicaciones con PIC, Marcombo, 2007.

34. W. Jensen, "The symbol for $\mathrm{pH} "$, Journal of Chemical Education, vol. 81, p. 21, 2004. 